\title{
TRANSVERSE IMPEDANCE IMPLEMENTATION IN ORBIT
}

\author{
V.Danilov, J. Galambos, J. Holmes, ORNL SNS Project, Oak Ridge, TN
}

\begin{abstract}
The transverse stability and broadening of the beam turn out to be the most problematic issues in the Spallation Neutron Source ring operation. The particle simulation code ORBIT, initially written for space charge and halo growth studies, now is able to cover a broad spectrum of the accumulator ring problems. Here we present an implementation of a collective force simulation due to impedance elements in the vacuum chamber of the ring.
\end{abstract}

\section{INTRODUCTION}

ORBIT is a new code developed for the Spallation Neutron Source (SNS) project. It includes $\mathrm{H}^{-}$injection modelling features needed to simulate realistic injection scenarios. As the SNS project will have an intense ( $\left.10^{14}\right)$, low energy beam $(1 \mathrm{GeV})$ and is concerned with keeping uncontrolled losses to $\sim 1$ part in $10^{4}$, space charge models are provided to calculate beam halo. More detailed descriptions on using the code are given in [1]. Here we focus on the impedance module.

The longitudinal impedance in ORBIT was implemented several years ago. It was based on an algorithm presented in [2], and uses products of Fourier coefficients of the current and the impedances at corresponding frequencies to calculate the longitudinal kick.

The transverse impedance implementation is based on the same approach. The complication is that the betatron motion has much higher frequency and the harmonics of the dipole current consist of the betatron sidebands of the revolution harmonics. Also, the number of transverse dimensions is two. Therefore the transverse impedance requires four times as many arrays and calculations than the longitudinal impedance.

\section{GENERAL SCHEME}

In the transverse collective phenomena code, the kicks are taken to be delta-functions, which is valid when the betatron phase advance over the physical extent of the impedance is small. Otherwise the impedance should be split into several small pieces, which is valid when the communication between pieces is negligible. If this is not the case, then a more general Green function approach, which is beyond the scope of the present paper, must be used.

The value of an element's impedance is passed into ORBIT numerically as an argument of the Impedance node. To calculate the transverse collective kick, we expand the function $\Psi$, which is the local product of the dipole moment and the current, at the position of the impedance into series of harmonics, and, multiplying them by the corresponding impedance, convert the harmonic series into a series for the kick. Since the dipole moment is not a periodic function of time, but has a betatron phase advance after the revolution period, we use the following formula for the "dipole moment times current" value:

$$
\begin{aligned}
\Psi(t) & =\cos \left(\omega_{b} t\right) f_{1}(t)+\sin \left(\omega_{b} t\right) f_{2}(t) \\
& =\cos \left(\omega_{b} t\right) \sum_{n=-N t o t+1}^{N t o t} a_{n} e^{i n \omega_{0} t}, \\
& +\sin \left(\omega_{b} t\right) \sum_{n=-N t o t+1}^{N t o t} b_{n} e^{i n \omega_{0} t}
\end{aligned}
$$

where $f_{1}, f_{2}$ are periodic functions of time (to be determined), $\omega_{0}$ and $\omega_{\mathrm{b}}$ are the revolution and the betatron frequencies, respectively, and $\mathrm{N}_{\text {tot }}$ is the total number of harmonics considered. The lower index in the sum is incremented by one because the fast Fourier transform should have even number of harmonics.

To determine $f_{1}, f_{2}$ functions, we assume that the collective, as well as the other perturbations, are small. In this case the dipole moment after one revolution transforms as a betatron coordinate through the Twiss parameters $\alpha, \beta$ and phase advance $\mu$ at the impedance point, and equals

$\Psi\left(t+T_{0}\right)=(\cos \mu+\alpha \sin \mu) \Psi(t)+\sin \mu \beta \Psi^{\prime}(t)$

Equating $\Psi$ to the Formula 1 at two times $t$ and at $t+T_{0}$, one obtains the expressions for the two coefficients $f_{1}, f_{2}$ :

$$
\begin{aligned}
& f_{1}(t)=\Psi(t)(\cos (\varphi)-\alpha \sin (\varphi))-\beta \Psi^{\prime}(t) \sin (\varphi), \\
& f_{2}(t)=\Psi(t)(\sin (\varphi)+\alpha \cos (\varphi))+\beta \Psi^{\prime}(t) \cos (\varphi) .
\end{aligned}
$$

where $\varphi=\omega_{b} t$. The coefficients $\mathrm{a}_{\mathrm{n}}, \mathrm{b}_{\mathrm{n}}$ in Formula 1 are just Fourier coefficients of the functions $f_{1}, f_{2}$, respectively.

The change in the horizontal or vertical angle is (see e.g. [3]):

$$
\begin{aligned}
& \Delta x^{\prime}\left(y^{\prime}\right)=\frac{\int F_{\perp} d s}{\beta^{2} E}=\frac{\operatorname{Re} i \sum_{i=-N b i n s+1}^{N b i n s} \Psi\left(\omega_{i}\right) Z_{\perp}\left(\omega_{i}\right)}{\beta^{2} E(e V)}= \\
& \frac{-\operatorname{Im} \sum_{n=-N b i n s+1}^{N b i n s} Z_{\perp}\left(n \omega_{0}+\omega_{b}\right)\left(a_{n}-i b_{n}\right) e^{i n \omega_{0} t+i \omega_{b} t}}{2 \beta^{2} E(e V)}- \\
& \frac{\operatorname{Im} \sum_{n=-N b i n s+1}^{N b i n s} Z_{\perp}\left(n \omega_{0}-\omega_{b}\right)\left(a_{n}+i b_{n}\right) e^{i n \omega_{0} t-i \omega_{b} t}}{2 \beta^{2} E(e V)}
\end{aligned}
$$




\section{HALO GENERATION DUE TO COLLECTIVE FIELD.}

Consider a coasting beam case with Lorentz energy distribution for a constant focusing storage ring and for single harmonic impedance. There is special interest in this problem. First, we can solve it exactly and check how the distribution evolves for all the particles and how the beam emittance and halo depends on the intensity (this is the main question for systems like the Spallation Neutron Source ring since it is directly related to its activation). Second, exactly solvable models give an excellent opportunity to check the accuracy of numerical methods and their predictive abilities.

We take the dipole moment $D$ of the particles at each point in the form $D=A \cos \left(\psi+\omega_{b} t\right)$, where A and $\psi$ are slow amplitude and phase, respectively. Since the collective force $F$ is only a small perturbation to the betatron motion, it is more convenient to use averaged equations. The oscillatory equation $D^{\prime \prime}+\omega_{b}^{2} D=F / m$ transforms into (see e.g. [4]):

$$
\frac{d A e^{i \psi}}{d \tau}=-i \frac{\beta_{f} \Pi}{2 m v^{2}} \int_{0}^{N} F \exp \left(-i \omega_{b} \tau \Pi / v\right) \frac{d \tau}{N},
$$

where $\beta_{\mathrm{f}}$ is the beta-function, $\tau$ is the new variable equal to $s / \Pi, \Pi$ is the circumference, $N(>>1)$ is a number of turns for averaging. $\mathrm{F}$ must be real in this equation. Let's take only one harmonic $\mathrm{n}$ with $D=\operatorname{Re} A \exp \left(i n \omega_{0}(t-\right.$ $\left.s / v)+i \omega_{b} t+i \varphi\right)$, ( $\psi$ for (3) equal to $\left.\psi=n \omega_{0}(t-s / v)+\varphi\right)$ the localised force is $F_{\perp}=e \delta_{\Pi}\left(s-s_{0}\right) \operatorname{Re} i I D Z_{\perp}\left(\omega_{b i}+n \omega_{0}\right)$, where $I$ is the current, $s_{0}$ is the position of the impedance in the ring, $\delta_{\Pi}$ is the periodic delta function with the period $\Pi$. After substitution into (3) one gets:

$$
\frac{d D_{s}}{d \tau}=\frac{|e| \beta_{f} I Z_{\perp}\left(n \omega_{0}+\omega_{b}\right)}{2 y m v^{2}} D_{s},
$$

where $\mathrm{D}_{\mathrm{s}}$ is the slow dipole moment, $\mathrm{D}_{\mathrm{s}}=$ $A \exp \left(i \psi+i \omega_{b} t\right)$. To obtain the final equation, one has to introduce dependence of the $\mathrm{D}_{\mathrm{s}}$ on the energy offset $\delta=\Delta E / E$, which just means replacement of the $D_{s}$ by the integral over the energy distribution function $\mathrm{g}(\delta)$, with its total integral equal to 1 .

$$
\frac{d D_{s}}{d \tau}=\frac{|e| \beta_{f} I Z_{\perp}\left(n \omega_{0}+\omega_{b}\right)}{2 m v^{2}} \int_{-\infty}^{\infty} D_{s} g(\delta) d \delta .
$$

Now we transform the full derivative with respect to dimensionless time $\tau$ into partial derivatives with respect to (again) $\tau$ and the relative position of particles $\mathrm{z}^{1}$ :

$$
\frac{\partial D_{s}}{\partial \tau}+z^{\prime} \frac{\partial D_{s}}{\partial z}=\chi \int_{-\infty}^{\infty} D_{s} g(\delta) d \delta
$$

\footnotetext{
1 the derivative with respect to the energy is absent because we deal with the coating beam and the energy change is equal to zero
}

where $\chi=\frac{|e| \beta_{f} I Z_{\perp}\left(n \omega_{0}+\omega_{b}\right)}{2 \gamma m v^{2}}$. The relative position $\mathrm{z}=\Delta \mathrm{s} / \Pi(\Delta \mathrm{s}$ is the distance between particles) derivative with respect to $\tau$ is $z^{\prime}=\eta \delta / \beta^{2}$ ( $\eta$ is the opposite sign slippage factor), and the dependence of $D_{s}$ on $z$ is fixed (for our particular harmonic) $D_{s}=d_{s} \exp (i 2 \pi n z)$, where $d_{s}$ is part of the slow dipole moment, which depends only on time and energy ${ }^{2}$. To keep the above equations simple we assumed $\omega_{b}$ doesn't depend on energy offset. The addition (for taking the energy dependence into account) is straightforward - it requires an addition of a linear dipole term in all the equations (36 ), with a coefficient, proportional to the energy offset and $\omega_{b}$ energy derivative. After substitution of the dipole moment into (6) we have:

$$
\frac{\partial d_{s}}{\partial \tau}+i \Delta d_{s}=\chi \int_{-\infty}^{\infty} d_{s} g(\delta) d \delta
$$

where $\Delta=\frac{2 \pi \eta \delta\left(n+v_{b}\right)}{\beta^{2}}$. The term with $v_{\mathrm{b}}$ in the force appears because particles' betatron phases change due to difference in velocities and the spatial chromaticity equals zero $\left(\partial v_{b} / \partial \delta=0\right.$, see also footnote (2)). If $g(\delta)=\frac{\delta_{0}}{\pi\left(\delta^{2}+\delta_{0}^{2}\right)}$, and the initial $\mathrm{d}_{\mathrm{s}}(\delta)=1$ the solution for (7) is:

$$
\begin{aligned}
& d_{s}=\exp (-i \Delta \tau)- \\
& \frac{\chi\left(\exp \left(-\left(\Delta_{0}-\chi\right) \tau\right)-\exp (-i \Delta \tau)\right)}{\Delta_{0}-\chi-i \Delta}
\end{aligned}
$$

where $\Delta_{0}=\frac{2 \pi \eta \delta_{0}\left|n+v_{b}\right|}{\beta^{2}}$.

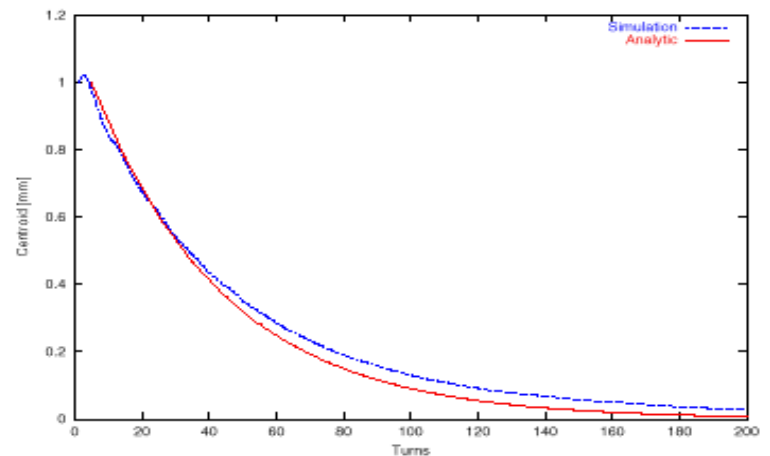

Figure 1 Simulated (blue) and analytical (red) dipole moment versus number of turns.

Figure 1 shows the ORBIT numerical and the analytical solutions from (8) for the centroids of one very short

\footnotetext{
${ }^{2}$ for zero impedance $d_{s} \sim \exp \left(i 2 \pi \eta \delta v_{b} / \beta^{2}\right)$ because even for zero chromaticity particles with different energies have different phase advance over same! Time because of difference in velocities. Similar phase change appear due to chromaticity.
} 
longitudinal slice. The parameters for comparison are the following. The lattice has constant beta function, only vertical motion has the impedance (for the case shown it is equal to $1 \mathrm{MOhm} / \mathrm{m}$ ). The total length of the ring is 248 meters, the frequencies are 6.4 (horizontal) and 6.3 (vertical), the number of protons is equal to $10^{14}$. In the simulation only particles with up to 10 Lorentz tune spreads were considered. The kinetic energy is $1 \mathrm{GeV}$, and the Lorentz energy spread is $0.01 \mathrm{GeV}$.

Equating $\chi$ to $\Delta_{0}$ we get the threshold impedance $Z_{\text {th }}=\frac{4 \pi \gamma \eta \delta_{0} m c^{2}\left|n+v_{b}\right|}{e \beta_{f} I}$, which is equal to 1.68 $\mathrm{MOhm} / \mathrm{m}$ for $\mathrm{n}=0$. The numerical runs give threshold values of about 1.6 MOhm/m, therefore the values of the threshold are within $5 \%$.

Figures 2 gives the comparison of the analytical solution (8) and the ORBIT simulation for rms beam size, divided by the beta function, (Figure 2, left). One can see that the size grows and saturates when the dipole oscillations disappear. It is interesting to get the ratio (we call it R) of the saturated rms size and rms size for zero intensity to see the factor of beam expansion. To get it, one has to take the squared real part of expression (8), integrate it with the Lorentzian distribution function, divide the result by one for zero intensity and take the square root of it. The final expression is:

$R=\frac{\sigma(\zeta)}{\sigma(0)}=\sqrt{\frac{\delta_{0}}{\delta_{0}-\zeta}}$ where $\delta_{0}$ is the parameter of the Lorentz energy distribution (see its definition after the equation (7)) and $\zeta=\frac{|e| \beta_{f} I Z_{\perp}\left(n \omega_{0}+\omega_{b}\right) \beta^{2}}{4 \pi m v^{2} \eta\left|n+v_{b}\right|}$. Since

$\zeta$ depends on current and $\delta_{0}$ determines the threshold, the expression for $\mathrm{R}$ could be more conveniently expressed via number of particles $\mathrm{N}$ and the threshold particle number $\mathrm{N}_{\text {th }}$ :

$$
\frac{\sigma(N)}{\sigma(0)}=\sqrt{\frac{N_{t h}}{N_{t h}-N}} .
$$

The parameter $\mathrm{R}$ of the size expansion is valid when we have narrow band impedance (for only one harmonic) and works only below the instability threshold.
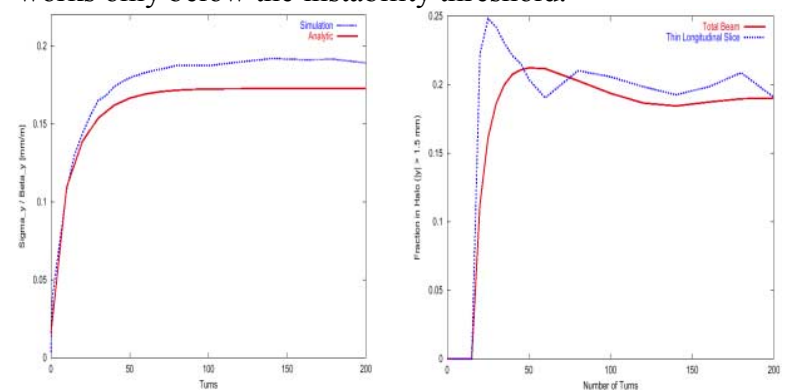

Figure 2. Numerical (blue) and analytic solutions for the rms size (left) and fraction of particles (right) with the ycoordinate larger than 1.5 initial displacement.
Figure 2, on the right, shows the fraction of particles with the vertical coordinate greater than 1.5 times the initial displacement. One can see that numerical solution gives about $10 \%$ larger result both for emittance and halo growth. This may be related to the finite longitudinal size of the slice (contrary to infinitely short slice in analytical solution), or to numerical noise.

The Figure 3 shows the vertical phase space of one short longitudinal slice. One can see that the simulation preserves even small details of an analytical solution, showing the ability of the code to give accurate results.
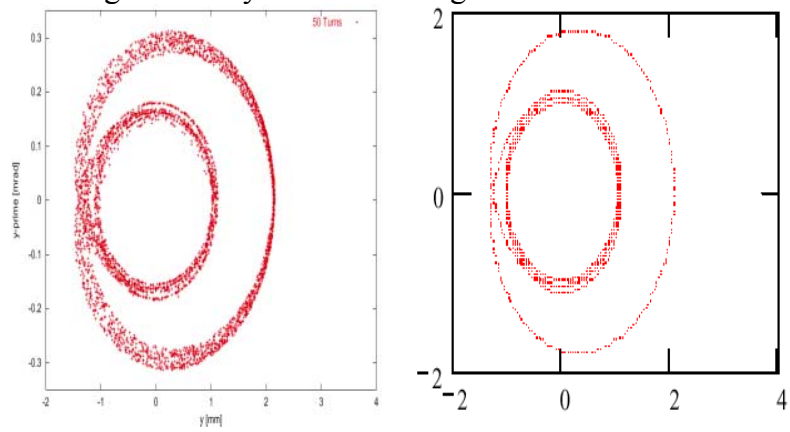

Figure 3. Phase space after 50 turns. Numerical solution on the left shows group of particles within short slice. The analytical solution (right) represents infinitely small size and is less fuzzy.

\section{CONCLUSION}

The SNS simulation code ORBIT was expanded with the capability to deal with transverse collective fields. It shows good agreement with the exactly solvable models for the transverse instabilities.

A new effect (from well-known Landau solutions for the Vlasov equation) was found. The large halo grows because of the collective fields, which transform initial displacement into a beam size, even if the intensity is well below the threshold.

\section{ACKNOWLEDGMENTS}

The authors thank A. Shishlo and J. Wei for the help and interest in the work. Research sponsored by the DOE, under contract no. DE-AC05-00OR22725with UTBatelle, LLC for ORNL

\section{REFERENCES}

[1] J. Galambos, et al, "ORBIT - a Ring Injection Code with Space Charge", PAC 99, New York, 1999.

[2] J.A. MacLachlan, "Longitudinal Space Tracking with Space Charge and Wall Coupling Impedance", FNAL TechNote, FN-446, February (1987).

[3] Chao, "Physics of Collective beam Instabilities", John Wiley \& Sons, Inc. (1993) p.69

[4] V. Migulin et al, "Osnovy teorii kolebaniy", Nauka, Moscow (1988) in Russian 